\title{
Functional liver-image guided hepatic therapy (FLIGHT): A technique to maximize hepatic functional reserve
}

Yukie Furukawa, B.S., C.M.D., R.T. (T) ${ }^{1}$, David E. Long, M.D. ${ }^{1}$, Susannah G. Ellsworth, M.D. ${ }^{1}$

1. Department of Radiation Oncology, Indiana University School of Medicine, Indianapolis, IN 46202, USA

Corresponding Author: Susannah G. Ellsworth, M.D. Department of Radiation Oncology, Indiana University School of Medicine, Indianapolis, IN 46202, USA (sgellswo@iu.edu)

\begin{abstract}
Introduction: Radiation planning approaches for liver radiation often do not consider the regional variation that can exist in liver function. This study dosimetrically compares functional liver image-guided hepatic therapy (FLIGHT) to standard stereotactic body radiation therapy (SBRT) plans. In the FLIGHT plans, functional data from hepatobiliary iminodiacetic acid (HIDA) single photon emission computed tomography (SPECT) scans serve as a road map to guide beam arrangement. While meeting the same target volume coverage, plans are optimized to reduce dose to high-functioning liver. Materials and Methods: The study included 10 patients with hepatocellular carcinoma (HCC) with baseline HIDA SPECT imaging. Standard SBRT plans which did not systematically incorporate these scans had previously been completed on all 10 plans. Retrospectively, FLIGHT plans were created based on the use of contours of relative liver function from
\end{abstract}


the HIDA SPECT as avoidance structures. Resulting dose to each relative functional liver structure was examined and compared qualitatively and using Wilcoxin rank-sum tests. Target coverage, doses to organs at risk (OARs), conformity index $(\mathrm{Cl})$, and gradient index (GI) were also evaluated. Results: While maintaining the same target coverage, FLIGHT plans reduced the mean dose to the high functioning liver by a median of $3.0 \mathrm{~Gy}$ (range 0.7 to $4.6 \mathrm{~Gy}$ ), which represented a $31.4 \%$ mean reduction compared to standard planning. FLIGHT plans reduced the volume of high functioning liver receiving 15 Gy by a mean of $59.3 \mathrm{cc}$ (range 7 to $170 \mathrm{cc}$ ), for a mean reduction of $41.9 \%$. The mean dose to areas of liver function defined by $25 \%$ to $100 \%$ and $50 \%$ to $100 \%$ maximum was reduced with FLIGHT from 10.5 Gy to 8.5 Gy and from 10.5 Gy to $7.5 \mathrm{~Gy}$, respectively ( $p<0.005$ for both comparisons). The FLIGHT plans' mean $\mathrm{Cl}$ and GI did not differ significantly from the standard plans' ( $p=0.721$ and 0.169 , respectively). Conclusion: FLIGHT SBRT allows for field design and plan optimization individualized to a patient's baseline regional liver function to maximize hepatic functional reserve. This personalized approach is achieved without compromising target coverage or OAR sparing.

Keywords: Hepatocellular carcinoma, Stereotactic body radiation therapy, Liver function 


\section{INTRODUCTION}

Liver malignancies, especially hepatocellular carcinoma, often arise in patients with cirrhosis, who can exhibit significant variability in their liver function. The primary methods of assessing the severity of cirrhosis are clinical scoring systems such as the Child-Turcotte-Pugh (CTP), modified end-stage liver disease, and albumin-bilirubin scores. The gold standard lab test for global liver function evaluation is indocyanine green retention at 15 minutes (ICG15). A main limitation of all these tests is the lack of information that they provide regarding regional variations in hepatic function within the liver of an individual patient, which are increasingly well described. The use of functional imaging in radiation planning approaches is not a new concept and has been applied in a number of disease sites, the earliest attempts being in lung radiation. ${ }^{123}$ Visualizing regional differences in hepatic function has become possible with functional imaging techniques such as technetium-99-mebrofenin (Tc99m) hepatobiliary iminodiacetic acid (HIDA) and Tc99m galactosyl human serum albumin scans, dynamic contrast enhanced (DCE) computed tomography (CT), and dynamic contrast enhanced magnetic resonance imaging, all of which have been validated to ICG15. ${ }^{45678}$ Expertise with different techniques can vary between institutions. At our institution, functional liver imaging has been done with HIDA scans, which provide a global liver function value as well as SPECT data, allowing a regional map of liver function to be generated.

Functional liver image-guided hepatic therapy (FLIGHT) incorporates this functional data to minimize dose to functional liver. With standard RT planning, liver function is assumed to be homogeneous and plans are optimized to meet certain dosevolume histogram constraints to avoid liver injury. ${ }^{9}$ Incorporating functional data in the 
planning process would be beneficial, especially in patients with poor baseline liver function where sparing of high functioning liver may reduce the risk of toxicity. This retrospective study was designed to investigate whether FLIGHT could result in better sparing of high functioning liver while maintaining comparable target coverage, organs at risk (OARs) sparing, and plan conformity compared to the standard SBRT plans.

\section{MATERIALS AND METHODS}

The study included patients with baseline HIDA scans who were treated with liver SBRT for hepatocellular carcinoma at a single institution between 2014 and 2017. For patient immobilization, either Pro-lok (CIVCO Radiotherapy, Coralville, IA) or Elekta (Elekta Oncology, Norcross, GA) stereotactic body frames were used. Abdominal compression was used as needed to keep diaphragm excursion below $1 \mathrm{~cm}$.

\section{RELATIVE FUNCTIONAL LIVER CONTOURING}

Each patient's treatment planning CT was rigidly fused with the baseline HIDA SPECT scan using MIM software (MIM Software Inc, Cleveland, OH). Liver function was divided into the following quartiles based on percent of maximum uptake within the liver structure: low ( $0 \%$ to $25 \%$ max), low-intermediate ( $25 \%$ to $50 \%$ max), high-intermediate ( $50 \%$ to $75 \%$ max), and high ( $75 \%$ to $100 \%$ ), and separate contours were created within the liver to encompass each functional level ( Figure 1 ). Contours corresponding to the higher levels of function were then used as avoidance structures for the FLIGHT plans. 

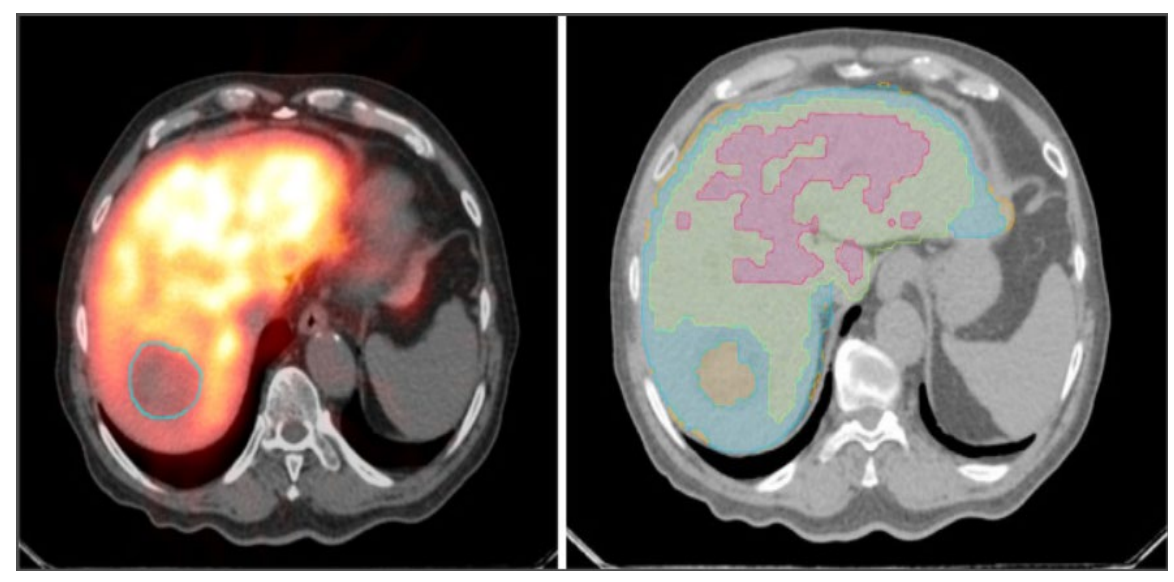

Fig. 1 SPECT HIDA scan fused to treatment planning CT (left) with contours of relative liver function based on HIDA SPECT scan (right). Liver function is represented by the following contours: low-intermediate (blue, $25 \%$ to $50 \%$ ), high-intermediate (green, $50 \%$ to $75 \%$ ), and high (magenta, $75 \%$ to $100 \%$ ). (Color version of figure is available online.)

\section{TARGET STRUCTURE CONTOURING}

The gross tumor volume (GTV) was contoured based on triple-phase CT, magnetic resonance imaging, or PET scans. To account for tumor motion due to patient breathing, the internal target volume (ITV) was delineated using four-dimensional (4D) CT. The planning target volume (PTV) was a $0.5 \mathrm{~cm}$ radial and 0.8 to $1 \mathrm{~cm}$ cranio-caudal expansion from the ITV to account for day-to-day set-up error. Identical target volumes were used for the FLIGHT and standard SBRT plans.

\section{TREATMENT PLANNING}

The HIDA SPECT guided plans were optimized so that the target coverage would be equivalent between the FLIGHT plan and standard SBRT plan. The plan objectives were to have $>98 \%$ of the GTV being covered by $110 \%$ of the prescribed dose, and to have $>95 \%$ of the PTV covered by $100 \%$ of the prescribed dose. All plans were inverse 
planned using Eclipse version 11.0.31 (Varian Medical Systems; Palo Alto, CA) with the anisotropic analytical algorithm (AAA). The beam energy (6MV or $18 \mathrm{MV})$ remained the same for all plans, but different geometric beam arrangements were used for the FLIGHT plans in order to maximize sparing of high functioning liver regions. Similar to typical liver SBRT planning, eight to ten non-coplanar, non-opposed fields were utilized for the HIDA SPECT guided plans. Figure 2 illustrates a sample FLIGHT plan, which has no beams entering from the left anterior oblique (LAO) angle in order to minimize entry dose to the high functioning portion of the liver, shown in the magenta contour.
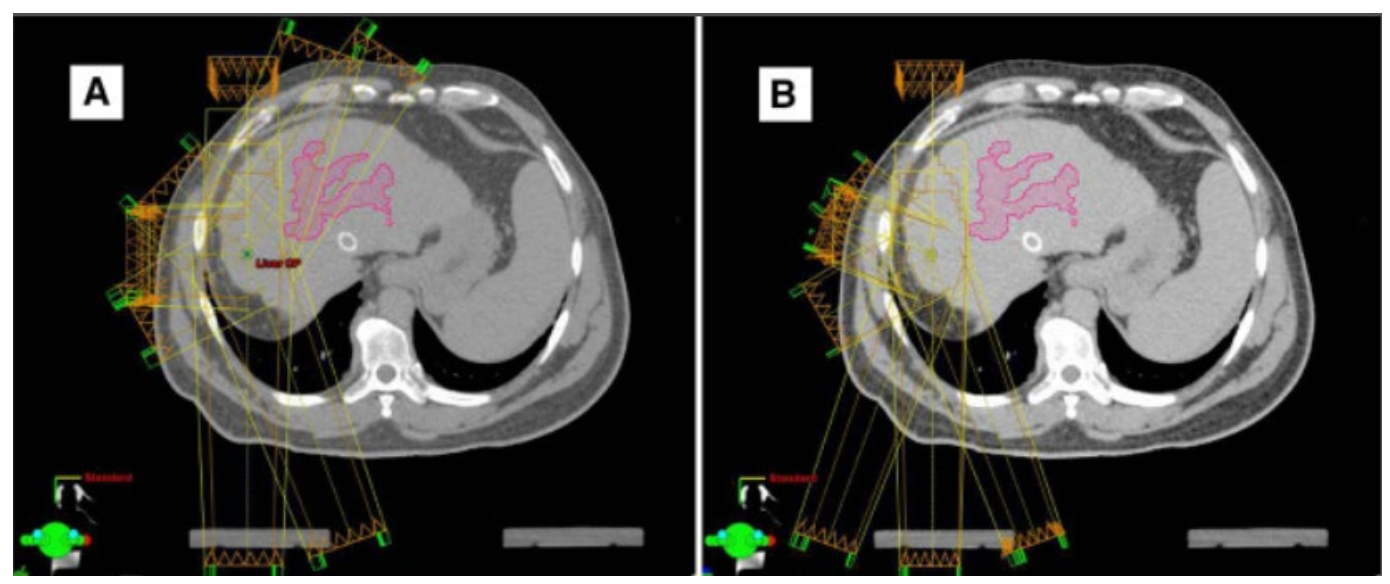

Fig. 2 Patient B: Beam arrangement of (A) standard SBRT plan, and (B) FLIGHT plan. High functioning liver ( $75 \%$ to $100 \%$ max) is shown in magenta. (Color version of figure is available online.)

\section{PLAN EVALUATION}

The resulting dose to each relative functional liver structure was compared between the FLIGHT and standard SBRT plans. To assess the effectiveness of high functioning liver sparing, mean dose and the volume receiving $\leq 15 \mathrm{~Gy}(\mathrm{~V} 15)$ to each relative functional liver structure were collected and compared between plans. GTV and PTV were not excluded from the recorded V15. Additionally, the conformity and integrity of the HIDA SPECT guided SBRT plans were assessed by target volume coverage, doses to OARs, 
conformity index $(\mathrm{Cl})$, and gradient index $(\mathrm{Gl})$. The $\mathrm{Cl}$ and $\mathrm{Gl}$ were calculated using the formulae given below: ${ }^{10}$

$$
\begin{gathered}
C I=\frac{P I(\text { theprescriptionvolume })}{P T V(\text { thetargetvolume })} \\
G I=\frac{P I 50 \%(50 \% \text { oftheprescriptionvolumeisodoseline })}{P T V(\text { thetargetvolume })}
\end{gathered}
$$

\section{STATISTICAL ANALYSES}

All statistical analyses were performed using IBM SPSS Statistics v24. The following parameters were compared between FLIGHT and standard SBRT using Wilcoxin ranksum tests: mean dose to relative functional liver structures, conformality index and gradient index.

\section{RESULTS}

Ten patients who had baseline HIDA scans prior to SBRT were included in this analysis. Patient characteristics were as follows: 3 women and 7 men, aged 56 to 77 years, 7 with CTP Class A cirrhosis and 3 with CTP Class B. The prescriptions were as follows: 48 Gy in 3 fractions $(n=5), 50$ Gy in 5 fractions $(n=2)$, and 40 Gy in 5 fractions $(n=3)$.

\section{DOSE TO THE RELATIVE FUNCTIONAL LIVER STRUCTURES}

Beam arrangements were changed in the FLIGHT plans to avoid the highest functioning liver in all 10 cases. Figure 3 demonstrates the difference in dose distribution that can be achieved with FLIGHT planning. The 15 Gy isodose line in the FLIGHT plan ( Fig. 3 B) clearly avoids the area of high functioning liver which had received low doses of radiation in the standard plan ( Fig. $3 \mathrm{~A}$ ). 

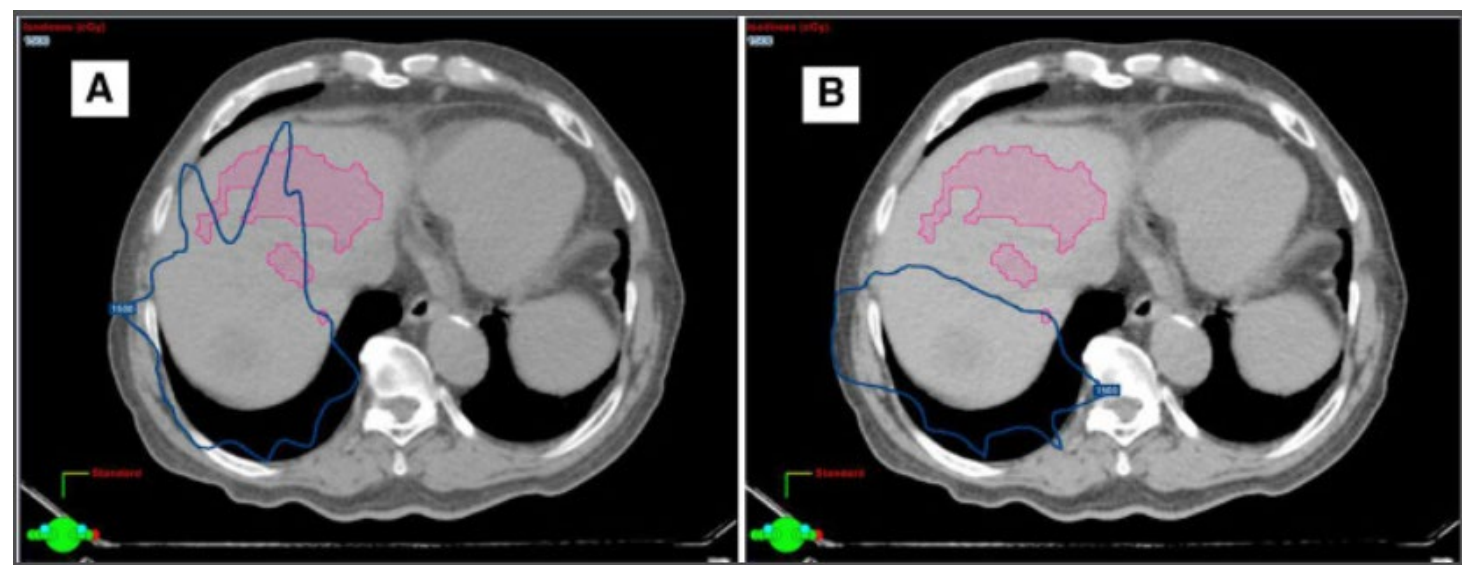

Fig. 3 Patient C: 15Gy isodose displayed for (A) standard SBRT plan, and (B) FLIGHT plan.

The mean dose to the functioning liver (25\% to $100 \%)$ was reduced by a mean of $20.1 \%$, and the same parameter for the high functioning liver (50\% to $100 \%)$ was reduced by a mean of $31.4 \%$. The median absolute reduction in dose to functioning liver ( $25 \%$ to $100 \%)$ was 2.0 Gy (range 0.6 to $2.9 \mathrm{~Gy}$ ) and the median reduction in dose to high functioning liver (50\% to $100 \%$ ) was 3.0 Gy (range 0.7 to $4.6 \mathrm{~Gy}$ ). After adjusting all prescriptions 50 Gy so that absolute mean doses could be compared, the mean dose to functioning liver was reduced with FLIGHT planning ( $p<0.005$ for both comparisons, Table 1 ).

Table 1

\begin{tabular}{|l|l|l|l|}
\hline & Standard & FLIGHT & $p$ Value \\
\hline Mean dose to 25\% to 100\% max functioning liver (+/- stdev) & $\begin{array}{l}10.5 \mathrm{~Gy} \\
(+/-4.4 \mathrm{~Gy})\end{array}$ & $\begin{array}{l}8.5 \mathrm{~Gy} \\
(+/-3.8 \mathrm{~Gy})\end{array}$ & 0.005 \\
\hline Mean dose 50\% to 100\% max functioning liver (+/- stdev) & $10.5 \mathrm{~Gy}$ & $7.5 \mathrm{~Gy}$ & 0.005 \\
\hline & $(+/-5.4 \mathrm{~Gy})$ & $(+/-4.5 \mathrm{~Gy})$ & \\
\hline
\end{tabular}


The percent of the volume of $25 \%$ to $100 \%$ functioning liver receiving 15 Gy was reduced by a mean of $21.9 \%$, and the same parameter for the $50 \%$ to $100 \%$ functioning liver was reduced by a mean of $41.9 \%$. The mean absolute volume reduction of structures receiving $>15$ Gy was $77.4 \mathrm{cc}$ for $25 \%$ to $100 \%$ functioning liver (range -6 to $176 \mathrm{cc}$ ) and $59.3 \mathrm{cc}$ (range 7 to $170 \mathrm{cc}$ ) for $50 \%$ to $100 \%$ functioning liver.

\section{PLAN CONFORMITY AND SPARING OF OARS}

FLIGHT plans' Cl and GI were not significantly different from standard SBRT plans ( Table 2 ). There were 2 plans treated with $48 \mathrm{~Gy}$ in 3 fractions in which the volume receiving > 30 Gy was $>30 \mathrm{cc}$ with the standard plans. In these cases, the volume of chestwall receiving > 30 Gy with the FLIGHT plan was kept below that of the standard plan. In one of these cases, the coverage of the GTV and PTV had to be compromised due to proximity to the chestwall. The coverage of the GTV and PTV was kept at or above that delivered with the standard plans.

Table 2

\begin{tabular}{|c|c|c|c|}
\hline & Standard & FLIGHT & $p$ Value \\
\hline Conformality index & 1.04 & 1.02 & 0.721 \\
\hline Gradient index & 3.73 & 3.50 & 0.169 \\
\hline
\end{tabular}




\section{DISCUSSION}

FLIGHT planning is a technique for individualizing radiation plans based on a patient's organ function. By placing integral dose in non- or low-functioning areas of the liver, thus avoiding high-functioning liver, hepatic functional reserve will theoretically be maximized. This study demonstrated that FLIGHT planning with HIDA scans is feasible and spares higher-functioning liver that otherwise would have received dose had the level of function not been known. The University of Michigan previously demonstrated that liver function can change during and post-treatment, and suggested that adapting a plan to direct dose through worse functioning liver may be feasible. ${ }^{6}$

The benefit of FLIGHT planning is highly patient dependent, and certain patients may benefit more from this approach than others. This has been noted by the University of Washington as well in their differential hepatic avoidance radiation therapy approach using sulfur colloid scans. ${ }^{11}$ Some plans, as shown in Figure 3, did not use beams from all potential angles. Notably, there was no change in conformity or gradient indices as a consequence of this. There was significant variation between patients in the location and the volume of the high functioning liver. Six patients had a reduction in mean dose to the high functioning liver ( $50 \%$ to $100 \%$ ) of $\geq 3 \mathrm{~Gy}$. Some patients' high functioning liver was large and distal from the target volume, which made it easier to avoid. On the other hand, others were challenging to avoid due to the proximity to the target ( Fig. 4 ). The quality of the registration of the HIDA to the planning CT is important, especially in cases where areas of high function are close to the target. There can be limitations with a rigid fusion of the SPECT HIDA with the planning CT, especially in cases where significant abdominal 
compression is used in a tumor of the left lobe of the liver, for example. In practice, clinical judgment as to the priority of functional avoidance is needed.

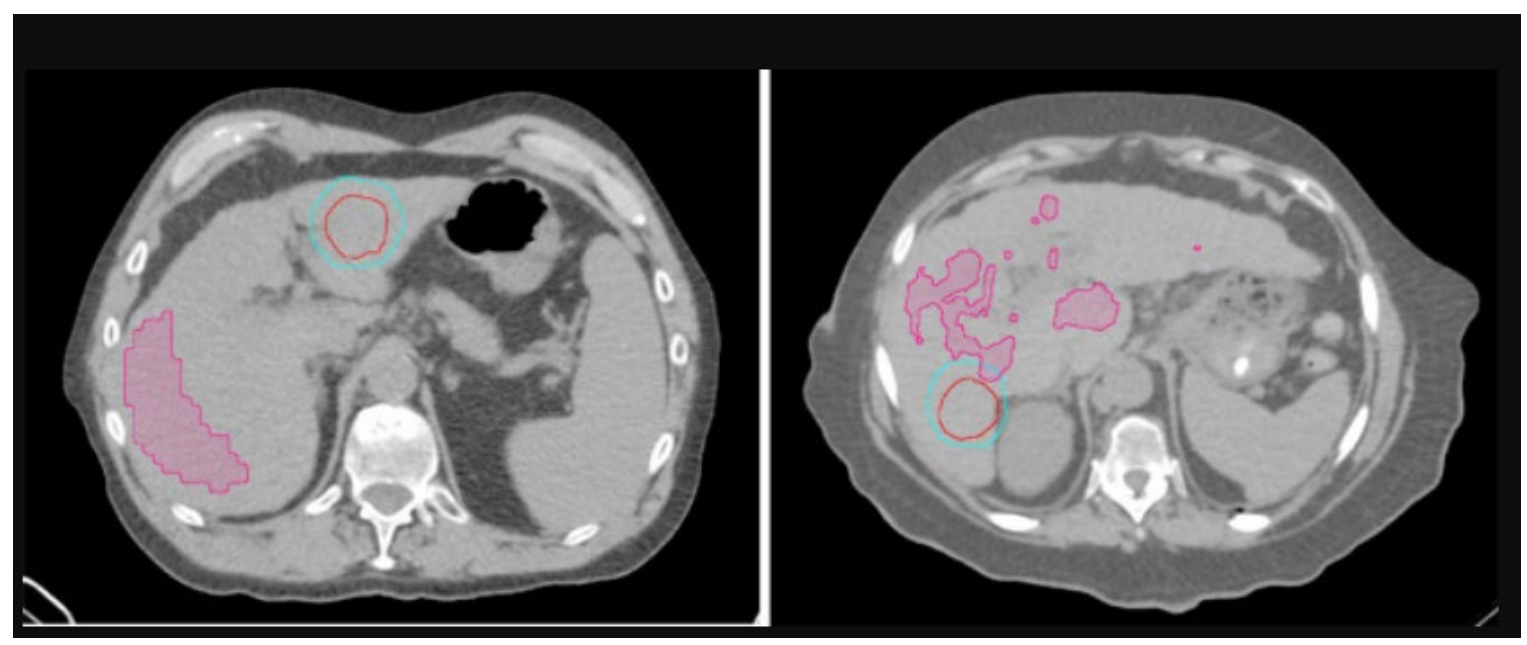

Fig. 4 Variation in the location and volume of the high functioning liver for Patient $D$ (left) and Patient E (right). GTV and PTV are shown in red and cyan contours, respectively. (Color version of figure is available online.)

The study was limited by sample size and its retrospective nature. The magnitude of the clinical benefit cannot be assessed by this dosimetric evaluation, but these preliminary results are encouraging. An analysis of post-treatment decompensation with the FLIGHT approach has been published, suggesting that functional dosimetric endpoints can complement current assessments of liver function. 12 This proof of concept approach formed the basis for the prospective FLIGHT study at our institution.

\section{CONCLUSION}

FLIGHT plans successfully spared functional liver while maintaining similar target coverage and conformity to conventional SBRT plans. The ability to spare functional liver without any measurable compromises demonstrates that FLIGHT planning is a 
reasonable approach, especially in patients with poor baseline liver function who may benefit from any incremental improvement in the plan. Evaluating FLIGHT prospectively may further elucidate its clinical benefit.

\section{Declaration of Competing Interest}

The authors declare that they have no known competing financial interests or personal relationships that could have appeared to influence the work reported in this paper.

\section{REFERENCES}

1. Marks, L. B., Spencer, D. P., Bentel, G. C., Ray, S. K., Sherouse, G. W., Sontag, M. R., Edward Coleman, R., Jaszczak, R. J., Turkington, T. G., Tapson, V., \& Prosnitz, L. R. (1993). The utility of SPECT lung perfusion scans in minimizing and assessing the physiologic consequences of thoracic irradiation. International Journal of Radiation Oncology*Biology*Physics, 26(4), 659-668. https://doi.org/10.1016/03603016(93)90285-4

2. Boersma, L. J., Damen, E. M. F., de Boer, R. W., Muller, S. H., Valdés Olmos, R. A., Hoefnagel, C. A., Roos, C. M., van Zandwijk, N., \& Lebesque, J. V. (1993). A new method to determine dose-effect relations for local lung-function changes using correlated SPECT and CT data. Radiotherapy and Oncology, 29(2), 110-116. https://doi.org/10.1016/0167-8140(93)90235-Z

3. Marks, L. B., Spencer, D. P., Sherouse, G. W., Bentel, G., Clough, R., Vann, K., Jaszczak, R., Coleman, R. E., \& Prosnitz, L. R. (1995). The role of three dimensional functional lung imaging in radiation treatment planning: The functional dose-volume histogram. International Journal of Radiation Oncology \&bull; Biology \&bull; Physics, 33(1), 65-75. https://doi.org/10.1016/0360-3016(95)00091-C 
4. Cao, Y., Pan, C., Balter, J. M., Platt, J. F., Francis, I. R., Knol, J. A., Normolle, D., Ben-Josef, E., Ten Haken, R. K., \& Lawrence, T. S. (2008). Liver Function After Irradiation Based on Computed Tomographic Portal Vein Perfusion Imaging. International Journal of Radiation Oncology ${ }^{\star}$ Biology ${ }^{*}$ Physics, 70(1), 154-160. https://doi.org/10.1016/j.ijrobp.2007.05.078

5. Cao, Y., Wang, H., Johnson, T. D., Pan, C., Hussain, H., Balter, J. M., Normolle, D., Ben-Josef, E., Ten Haken, R. K., Lawrence, T. S., \& Feng, M. (2013). Prediction of Liver Function by Using Magnetic Resonance-based Portal Venous Perfusion Imaging. International Journal of Radiation Oncology ${ }^{*}$ Biology ${ }^{\star}$ Physics, 85(1), 258263. https://doi.org/10.1016/j.ijrobp.2012.02.037

6. Wang, H., Feng, M., Frey, K. A., Ten Haken, R. K., Lawrence, T. S., \& Cao, Y. (2013). Predictive Models for Regional Hepatic Function Based on 99mTc-IDA SPECT and Local Radiation Dose for Physiologic Adaptive Radiation Therapy. International Journal of Radiation Oncology*Biology*Physics, 86(5), 1000-1006. https://doi.org/10.1016/j.jirobp.2013.04.007

7. Bennink, R. J., Dinant, S., Erdogan, D., Heijnen, B. H., Straatsburg, I. H., Vliet, A. K. van, \& Gulik, T. M. van. (2004). Preoperative Assessment of Postoperative Remnant Liver Function Using Hepatobiliary Scintigraphy. Journal of Nuclear Medicine, 45(6), 965-971.

8. Graaf, W. de, Bennink, R. J., Veteläinen, R., \& Gulik, T. M. van. (2010). Nuclear Imaging Techniques for the Assessment of Hepatic Function in Liver Surgery and Transplantation. Journal of Nuclear Medicine, 51(5), 742-752.

https://doi.org/10.2967/jnumed.109.069435 
9. Andolino, D. L., Johnson, C. S., Maluccio, M., Kwo, P., Tector, A. J., Zook, J., Johnstone, P. A. S., \& Cardenes, H. R. (2011). Stereotactic Body Radiotherapy for Primary Hepatocellular Carcinoma. International Journal of Radiation Oncology ${ }^{*}$ Biology ${ }^{*}$ Physics, 81(4), e447-e453. https://doi.org/10.1016/j.jijobp.2011.04.011

10.Shaw, E., Kline, R., Gillin, M., Souhami, L., Hirschfeld, A., Dinapoli, R., \& Martin, L. (1993). Radiation therapy oncology group: Radiosurgery quality assurance guidelines. International Journal of Radiation Oncology ${ }^{*}$ Biology ${ }^{\star}$ Physics, 27(5), $1231-$ 1239. https://doi.org/10.1016/0360-3016(93)90548-A

11. Bowen, S. R., Saini, J., Chapman, T. R., Miyaoka, R. S., Kinahan, P. E., Sandison, G. A., Wong, T., Vesselle, H. J., Nyflot, M. J., \& Apisarnthanarax, S. (2015). Differential hepatic avoidance radiation therapy: Proof of concept in hepatocellular carcinoma patients. Radiotherapy and Oncology, 115(2), 203-210. https://doi.org/10.1016/j.radonc.2015.04.011

12. Long, D. E., Tann, M., Huang, K. (Colin), Bartlett, G., Galle, J. O., Furukawa, Y., Maluccio, M., Cox, J. A., Kong, F.-M. (Spring), \& Ellsworth, S. G. (2018). Functional liver image guided hepatic therapy (FLIGHT) with hepatobiliary iminodiacetic acid (HIDA) scans. Practical Radiation Oncology, 8(6), 429-436. https://doi.org/10.1016/j.prro.2018.04.014 